\title{
Atlas V Aft Bulkhead Carrier Rideshare System
}

\author{
Maj Travis Willcox \\ NRO/OSL \\ 14675 Lee Rd Chantilly, VA 20151; 703-808-6676 \\ Travis.Willcox@nro.mil
}

\begin{abstract}
This paper gives the background and details of the Atlas V Aft Bulkhead Carrier to be flown on the National Recoinnassance Office Launch 36 with the Operationally Unique Technologies Satellite Auxiliary Payload. The CubeSats included are from a number of labs, universities and government entities for the purpose of technology demonstration, science experimentation and operational proof of concepts. This mission will pave the way for rideshare on NRO missions and other Atlas V launches.
\end{abstract}

\section{INTRODUCTION}

During the 1980s and 1990s, many small satellites were able to take advantage of the opportunity to be manifested on existing launches, particularly the Space Shuttle's Get Away Specials, commonly referred to as "gas cans". However, as the shuttle program suffered setbacks and became dedicated to International Space Station missions, these "rideshare" opportunities decreased significantly. Meanwhile, the Evolved Expendable Launch Vehicle (EELV) fleet was developed to launch the nation's very critical - and very expensive - national security satellites, but rideshare opportunities have not been a priority. Not surprisingly, program managers of primary spacecraft are not enthusiastic to add auxiliary payloads (APs). The net result, in their view, is reduced performance margin and some, however small, increase to mission risk.

The National Reconnaissance Office (NRO) has recognized, since its inception, that there are many efficiencies to be gained through rideshare in space launch, as well as many opportunities for the demonstration and application of emerging technologies through the use of small satellites. In 1999, the NRO's Office of Space Launch (OSL) hosted the first unclassified "Rideshare" Conference in Chantilly, VA dedicated to the small satellite community's concern about access to space. The conference was - and is - open to all government, industry, and academic entities interested in fostering rideshare opportunities for small satellites. While providing this forum, OSL also focused on the practice of manifesting smaller satellites on existing launches. The conference continues today and is held on an annual basis. Since 1999, OSL has investigated rideshare opportunities for the NRO's small satellites.
For the most part, that effort has been to track other agency's efforts to develop capabilities and opportunities. However, that recently changed with OSL's initiative to develop the Aft Bulkhead Carrier (ABC) for use on the Atlas V launch vehicle. OSL has completed the design of this system and will use it to launch 11 cubesats of various configurations on NRO Launch 36 (NROL-36), in the summer of 2012. This significant achievement will hopefully pave the way for AP's on many future NRO and other government launches.

\section{Design \& Development}

OSL partnered with United Launch Alliance (ULA), the Delta IV and Atlas V launch vehicle provider, to develop the $\mathrm{ABC}$ system, which will make use of the volume and structural capacity made available when ULA redesigned the Atlas $\mathrm{V}$ Centaur upper stage pressure system. This redesign replaced three spherical helium bottles with two larger cylindrical bottles, which left a volume of approximately $20 \times 30 \times 30$ inches available to attach up to a maximum of $220 \mathrm{lbs}$ to the aft bulkhead of the Centaur. ${ }^{*}$

Intuitively, the aft end of a vehicle's upper stage may not seem like a logical place to mount small spacecraft, but there are some advantages, particularly as they pertain to launching with an NRO primary satellite. These include:

1. All Auxiliary Payload (AP) operations take place off-line of the primary spacecraft activities

"For more information, see the ABC User's Guide to be published by the United Launch Alliance in 2012. 
2. Operations are done in parallel with the rest of the mission, causing no serial schedule impact

3. AP access to the primary payload volume is not required

4. All electrical signals are carried in cabling separate from the primary spacecraft signals

5. The ABC is not in the primary spacecraft load path
6. Compatibility assessments of the AP with the primary spacecraft are simplified, such as contamination and Electromagnetic Interference/Electromagnetic Compatibility (EMI/EMC) analyses

Figure 1. shows the location of the $\mathrm{ABC}$ in relation to the rest of the Atlas $\mathrm{V}$ launch vehicle and the primary spacecraft.

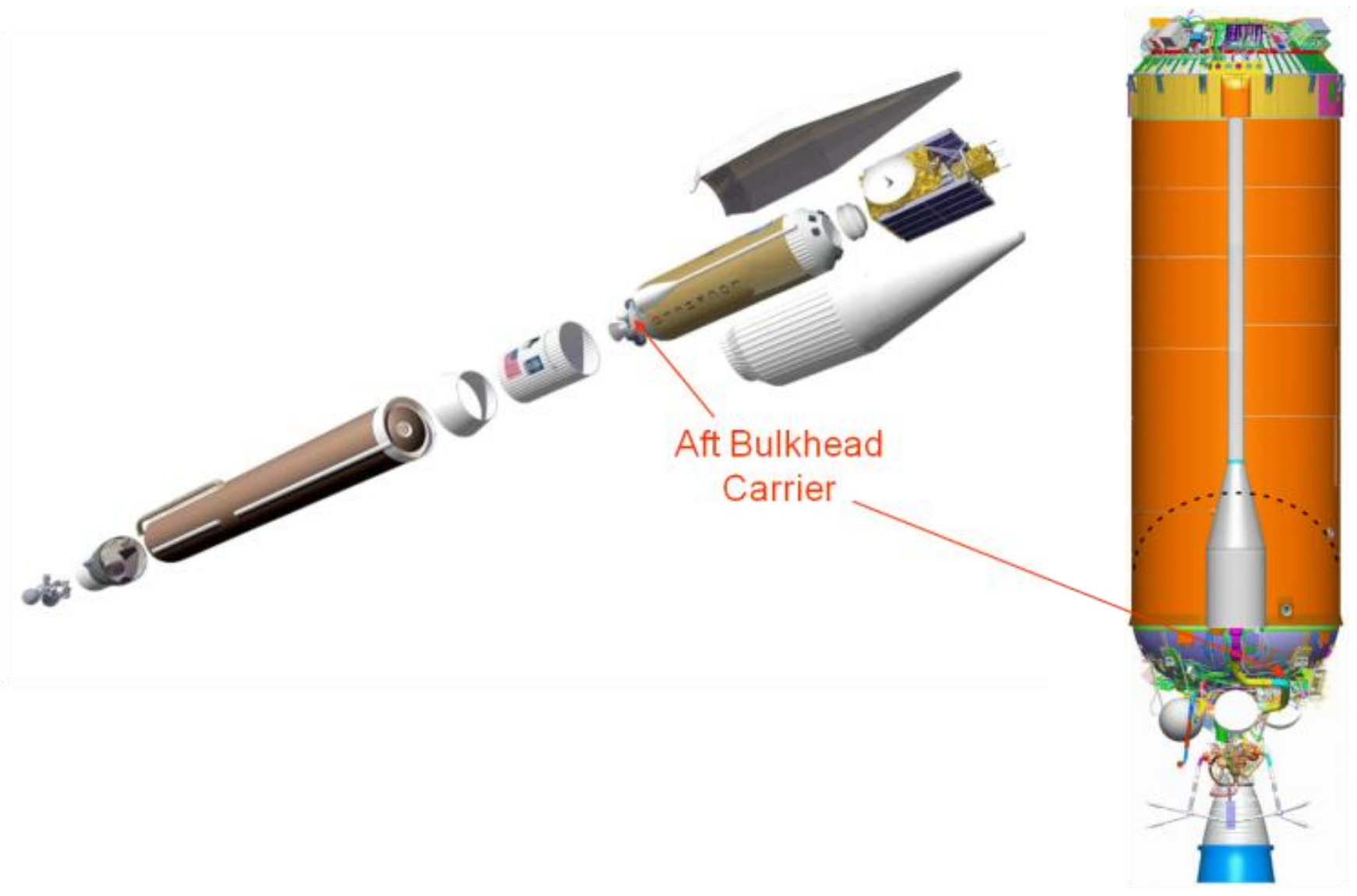

Figure 1: ABC on Atlas V Centaur

As OSL began this development, a number of groundrules were established to minimize (1) the cost of the effort, (2) the impact to the existing Centaur, (3) the impact to overall Atlas V build and launch site operations, and (4) the impacts to the primary spacecraft - all while maximizing the amount of payload the $\mathrm{ABC}$ could carry to orbit.

Numerous trades were conducted to accomplish these goals. To meet the first two goals, the criterian was established that no new structural testing of the ABC/Centaur aft bulkhead would be conducted. This meant $\mathrm{ABC}$ system had to be designed with a structural safety margin of at least 2.0. With that, calculations indicated a maximum ABC/AP system mass capability of $220 \mathrm{lbs}$. At first, the team was concerned that this constraint would significantly limit the AP mass to orbit. However, after numerous configurations were considered for the $\mathrm{ABC}$ system on all Atlas V variants, the volume was maximized and system complexity minimized with a design that resulted in the $20 \mathrm{x} 20 \mathrm{x}$ 30 inch volume previously mentioned. Using "typical" densities of recently flown smallsats, it became obvious that payloads exceeding $200 \mathrm{lbs}$ were unlikely candidates for this specific rideshare platform and the associated mission. Because of this, the design 
requirement for at least a 2.0 margin of safety was not overly constraining on the ABC's throw weight to orbit.

The ABC is a straightforward design made up of a plate and two struts that use the same four Centaur aft bulkhead attach fittings that were previously used for the spherical helium tank. Figure 2 illustrates the ABC design. This design results in a system that can accommodate an AP of up to $178 \mathrm{lbs}$ and provides the same loading conditions as the spherical helium bottle previously mounted the same location.

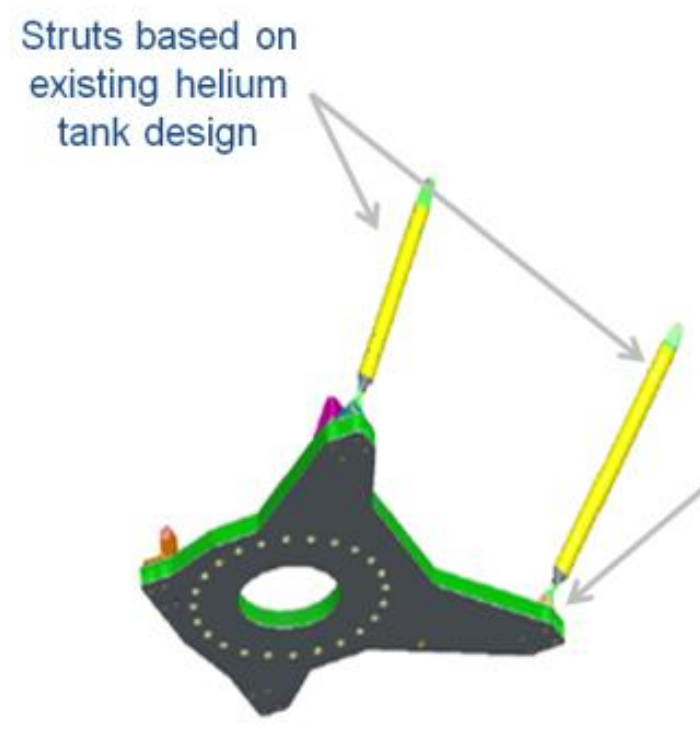

$A B C$ plate is of standard Atlas $\mathrm{V}$ material/construction - Aluminum facesheets with aluminum honeycomb core

Figure 2: ABC Plate and Struts

To minimize design and component testing costs, as well as maintain consistency with other Atlas structural components, the ABC plate uses a standard ULA aluminum honeycomb core/aluminum facesheet composite design found throughout the vehicle. ULA uses the same supplier to build the ABC plate that builds the other aluminum composite structures. In addition, the struts are of a design very similar to the struts used to mount the helium bottles and other such structures to the aft bulkhead. Therefore, the team was, again, able to simplify things by maintaining the existing supplier base.

Figure 3. illustrates the location of the $\mathrm{ABC}$ plate and the associated AP volume available. The "inboard" portion of the plate mounts directly to the aft bulkhead, while the "outboard" is attached using the two struts discussed above. The figure also depicts the two cylindrical helium bottles and the location of the ABC in relation to the RL-10 engine and its nozzle cone. The $\mathrm{ABC}$ is canted 17 degrees off the Centaur centerline. This solution was determined, after many iterations, to: maximize available volume; minimize loading on the $\mathrm{ABC}$ plate and struts; avoid impingement from venting of propellant, venting of pressure tanks and firing of thrusters; and maximize AP throw weight. The "notch" in the AP volume is to accommodate the separation dymanics of the Atlas/Centaur interstage adapter in the 4-meter payload fairing configuration, depicted in Figure 

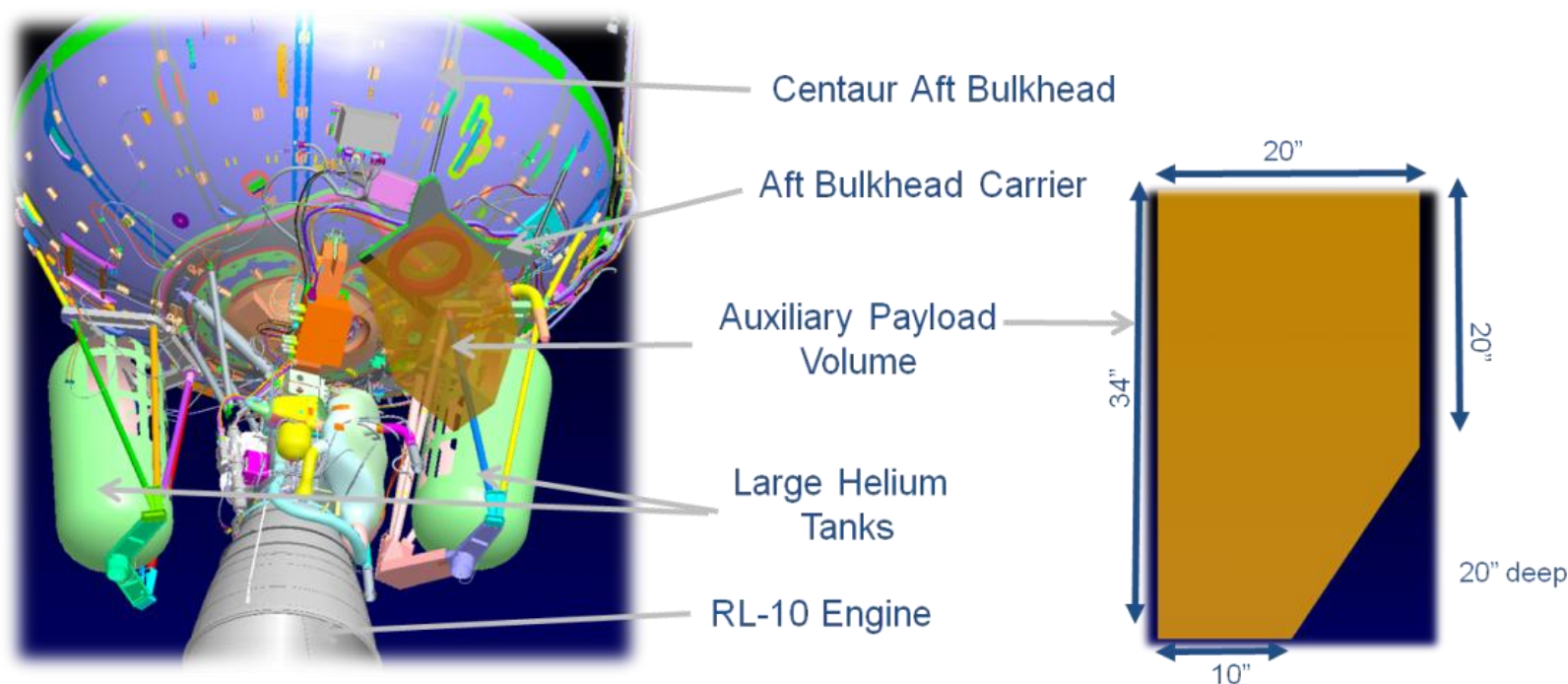

Figure 3: ABC Plate Mounted to aft End of Centaur Showing Volume Available for AP

\section{History}

Feasibility studies for ABC began in early 2008. The plan at that time was simply to hone in on a single concept and take it to Critical Design Review (CDR) level by 2010 . However, shortly after the feasibility study was initiated and prior to the Systems Requirements Review in July 2008, the NRO's cubesat office requested a "full-court press" to see if not only the design could be completed but a contingent of at least eight cubesats could be manifested as early as possible. A candidate mission, NROL-41 scheduled to launch in September 2010, was identified, and after completing a Preliminary Design Review (PDR) of the generic ABC design in December 2008, OSL focused all future $\mathrm{ABC}$ efforts on flying a specific NRO cubesat mission on NROL-41.

The mission was became known as AS\&T (Advanced Systems \& Technology) Development And Maturation Satellite (ADAMSat) and consisted of a combination of $1 \mathrm{U}, 2 \mathrm{U}$, and $3 \mathrm{U}$ cubesats in eight Poly-Picosat Orbital Deployers (P-PODs) built by the California Polytechnic University (Cal Poly). The eight P-PODs were mounted in a structure built by the Naval Postgraduate School (NPS) known as the NPS Cubesat Launcher - or NPSCuL (pronounced "NPS-cool"). OSL worked with the NROL-41 mission teams, the primary satellite customer, and Space and Missiles Center/Launch Range (SMC/LR), the Air Force agency responsible for procuring the NRO's launch vehicles, in order to get concurrence to proceed with the effort and understand the criteria for final approval to integrate ADAMSat. The schedule to make the NROL-41 launch was extremely aggressive, particularly since ADAMSat would have to be mated to the Centaur before it was stacked on the booster on the pad. For NROL-41, this meant ADAMSat had to be ready in April 2010. A super effort by ULA resulted in completion of the mission specific design, $\mathrm{CDR}$, analyses and $\mathrm{ABC}$ flight hardware build and installation on the NROL-41 Centaur by December 2009. Shortly afterwards, however, it became clear to the NRO cubesat office that ADAMSat would not be able to meet the April deadline and it was removed from the launch manifest. The removal of ADAMSat from NROL-41 was disappointing, but the foundation was laid for using the $\mathrm{ABC}$ for future rideshare opportunities. The efforts to work with the mission team, primary customer, and SMC/LR to foster a "can-do" attitude amongst all parties may be even more significant than the technical accomplishments made over the 20 -month period since the inception of $\mathrm{ABC}$.

OSL continued with the design of the generic $\mathrm{ABC}$ concept - capable of not only carrying the nonseparating NPSCuL, but also a single, separating smallsat. The effort culminated with the completion of a "generic" CDR in August of 2010. Whereas the mission-specific efforts for ADAMSat addressed the needs of that single launch, the generic design covered not only multiple AP configurations, but also a multitude of launch vehicle configurations, mission profiles, launch day operations and environments that encompassed all foreseeable rideshare mission candidates. The application of the generic rideshare platform will be determined for each individual mission (e.g.: single separating auxiliary payload, multiple cubesats, or any other configuration that can make use of the common interface on the $\mathrm{ABC}$ plate while meeting the mission and design constraints). Finally, a mini-pathfinder was conducted at Vandenberg Air Force Base (AFB) using a mockup of ADAMSat to demonstrate and validate the processes, procedures, and 
ground support equipment designed specifically for the installation of the ABC/AP system on the Centaur.

\section{The OUTSat Mission}

Although each mission will be different, the final orbit must achieve three key objectives: (1) adequate separation from the primary spacecraft orbit, (2) a useful orbit for the AP to accomplish its mission(s), and (3) an orbital lifetime fully compliant with space debris mitigation policies. With these objectives in mind, the decision was made, in August of 2010, to pursue launching another "ADAMSat-like" cubesat mission on NROL-36. The design and profile of this mission would provide favorable characteristics for placing cubesats in a desirable Low Earth Orbit after the deployment of the primary satellite and the final upper stage burn. The

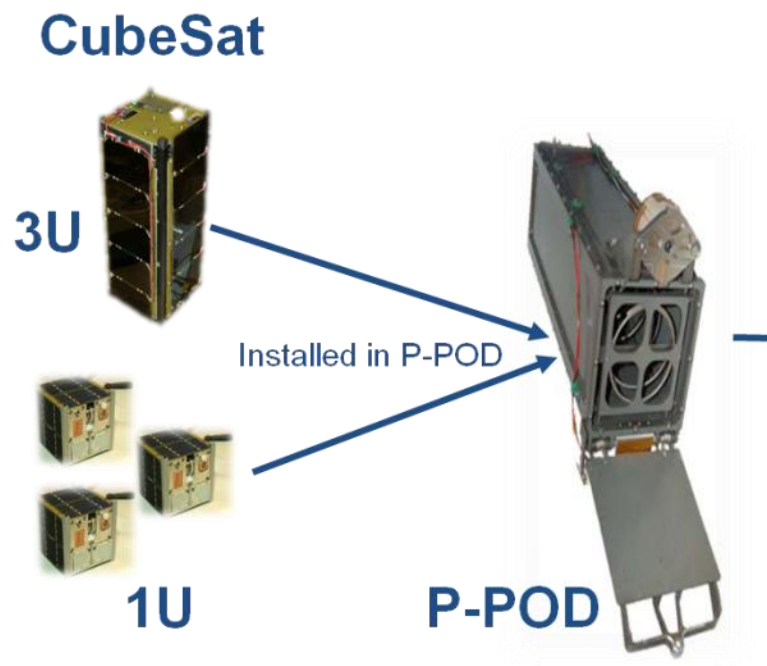

"satellite", now known as the Operationally Unique Technologies Satellite or OUTSat, came to consist of 11 cubesats in various configurations.

Figure 4. shows the OUTSat configuration. The eight PPODs each contain one to three cubesats. The integration was accomplished at Cal Poly's campus in San Luis Obispo, CA. The P-PODs were then mounted in the NPSCuL structure at NPS in Monterey, CA. Harnessing, not shown, was installed to provide (a) separation signals to the Non-Explosive Assembly (NEA) and (b) monitoring signals for the doors on each P-POD. These monitoring signals are used to monitor the open/closed status of the P-POD doors to ensure proper configuration for launch and provide verification to ground personnel that each door opens when the separation command is sent.

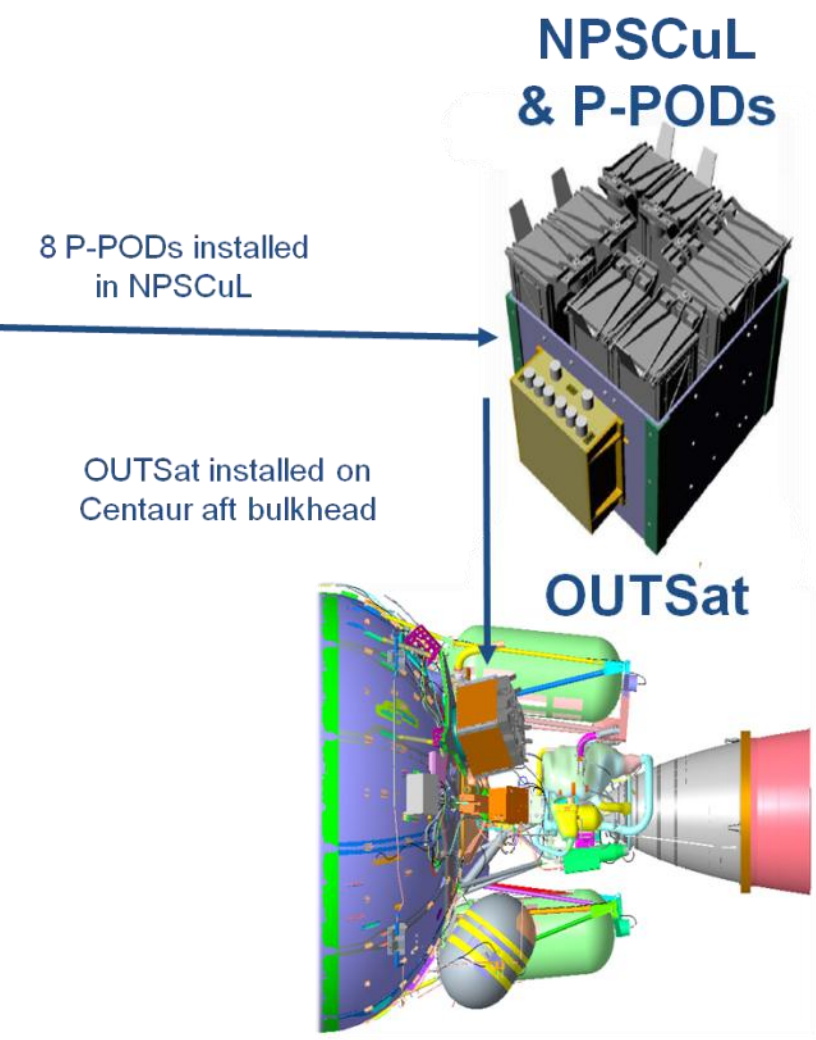

Figure 4: OUTSat configuration

The manifest for the mission, detailed in Table 1, was finalized after OUTSat integration and acceptance testing and consists of cubesats sponsored by the NRO's Mission Support Division (MSD) and NASA's Launch Support Program (LSP). The NASA cubesats are part of the ongoing Educational Launch of Nanosats (ELaNa) program to foster partnerships with educational institutions, providing students the opportunity to build and fly satellites, test emerging technologies, conduct scientific research, and demonstrate economical commercial off-the-shelf components that may be useful in future space missions.

One of the critical lessons learned from the NROL-41 effort was to ensure an adequate number of cubesats are ready to fly in case one or more cubesats are unable to deliver on schedule. For OUTSat, there were originally 16 cubesats on the list of candidates; at the time of integration, there were 14 remaining: 9 primary and 5 backup. However, thanks to the flexibility provided by this manifesting strategy, a late decision was made to 
replace one of the primary cubesats with a backup to allow for component rework. Table 1, below, shows the final manifest, while Table 2 provides the list of backup cubesats that were prepared to be integrated into the mission in case one of the primary cubesats could not be delivered in time.

Table 1: OUTSat CubeSat Manifest

\begin{tabular}{|c|c|c|c|c|c|}
\hline P-POD Position & Sponsor & Developer & CubeSat Name & Mission & Configuration \\
\hline 1 & NRO MSD & $\begin{array}{l}\text { Space and Missile Defense } \\
\text { Command }\end{array}$ & SMDC 1.2 & Comm & $3 \mathrm{U}$ \\
\hline 2 & NRO MSD & Aerospace Corporation & Aerocube & Smallsat Tech Demo & $3 \times 1 U$ \\
\hline 3 & NRO MSD & $\begin{array}{l}\text { University of Southern } \\
\text { California }\end{array}$ & AENEAS & GEO Tracking & $3 \mathrm{U}$ \\
\hline 4 & NASA LSP & University of Colorado & CSSWE & Space Weather & $3 \mathrm{U}$ \\
\hline \multirow[t]{2}{*}{5} & NASA LSP & Cal Poly & $\mathrm{CP5}$ & Debris Mitigation & $1 \mathrm{U}$ \\
\hline & & Morehead State University & CXBN & Space Weather & $2 \mathrm{U}$ \\
\hline 6 & NASA LSP & UC Berkeley & CINEMA & Space Environment & $3 \mathrm{U}$ \\
\hline 7 & NRO MSD & $\begin{array}{l}\text { Lawrence Livermore National } \\
\text { Laboratory (LLNL) }\end{array}$ & $\operatorname{Re}$ & Space Debris Mitigation & $3 \mathrm{U}$ \\
\hline 8 & NRO MSD & $\begin{array}{l}\text { Space and Missile Defense } \\
\text { Command }\end{array}$ & SMDC 1.1 & Comm & $3 \mathrm{U}$ \\
\hline
\end{tabular}

Table 2: Backup CubeSat Manifest

\begin{tabular}{|l|l|l|l|l|}
\hline \multicolumn{1}{|c|}{ Sponsor } & \multicolumn{1}{|c|}{ Developer } & \multicolumn{1}{|c|}{ CubeSat Name } & \multicolumn{1}{c|}{ Mission } & Configuration \\
\hline NRO MSD & $\begin{array}{l}\text { Space and Missile } \\
\text { Defense Command }\end{array}$ & SMDC 1.3 & Comm & $3 \mathrm{U}$ \\
\hline NRO MSD & $\begin{array}{l}\text { Space and Missile } \\
\text { Defense Command }\end{array}$ & SMDC 1.4 & Comm & $3 \mathrm{U}$ \\
\hline NRO MSD & $\begin{array}{l}\text { Air Force Institute of } \\
\text { Technology }\end{array}$ & ALICE & Space Weather & $3 \mathrm{U}$ \\
\hline
\end{tabular}




\section{Auxiliary Payload Integration Contractor (APIC)}

Another extremely important lesson learned from the ADAMSat effort was the need for a fully qualified integrating contractor that is on contract and engaged to support early in the program. This is especially critical for missions like OUTSat that bring a multitude of satellites to the mission. The APIC needs to not only be responsible for integrating the OUTSat and ensuring a fully tested satellite is delivered but also for integrating the AP with the launch vehicle, making sure all Range Safety, launch base, transportation, operations, and other documentation is complete and delivered on-time. In addition, the APIC is responsible for compiling all technical evidence to be presented to the primary spacecraft customer and the launch vehicle provider to demonstrate that including the $\mathrm{AP}$ on the launch is compatible with the existing mission and is of acceptable risk. In order to task a competent APIC in a timely fashion, OSL teamed with NASA's LSP to leverage their experience in manifesting cubesats and their existing contract with Cal Poly to provide such services. Using the NASA contract as a baseline and detailing the myriad of other requirements required to complete the integration effort, OSL was able to contract with Cal Poly in a timely fashion to provide APIC services for the OUTSat mission. Cal Poly teamed with SRI International as a subcontractor to provide a team with comprehensive skills to accomplish the seemingly endless tasks required to satisfy not only the requirements of OSL, but ULA, Range Safety, and the primary customer.

\section{Contingency/Simulator Approach}

Once ULA has designed a mission to accommodate the mass and dynamics of the OUTSat, it is imperative that what flies matches what was analyzed. One of the fundamental concerns of the primary customer and launch community is an unacceptably delinquent AP. Therefore, a contingency plan must be in place.

For the OUTSat mission, there are several "no show" scenarios. The first is if any number of individual $3 u$ cubesats do not show up. The second is if a $1 \mathrm{U}$ or $2 \mathrm{U}$ cubesat(s) does not make it. And finally, the situation where the entire OUTSat is a no-go. As addressed earlier, the primary contingency plan for the OUTSat mission is to have plenty of backup satellites to ensure that if either of the first two scenarios occur it is a simple "plug-nplay" of the backup. To date, this approach has been successful. However, if backup cubesats cannot be used, the following contingencies would be implemented:

1. If a 3U satellite is not available, the APIC must be on contract to provide a P-POD simulator that would be installed in the NPSCuL in lieu of the flight P$\mathrm{POD} /$ cubesat. The simulator would have weights added in the proper locations so that the total mass and center of gravity (cg) matches that of the replaced flight unit.

2. If either a $1 \mathrm{U}$ or $2 \mathrm{U}$ cubesat is not available, the APIC will need to provide a simulator of appropriate weight and cg to be secured with screws to the PPOD pusher spring, preventing deployment of the mass simulator on orbit. Note: The remaining flight $1 \mathrm{U}$ or $2 \mathrm{U}$ satellite(s) would be deployed when the PPOD door is opened.

In the case where the entire AP is not available, an appropriate mass simulator will be required. For OUTSat, OSL contracted with ULA to provide the mass simulator. OSL chose to contract with ULA and not the smallsat provider for several reasons:

1. It would be expensive for the smallsat or APIC community to provide a full-up simulator that replicated the flight article

2. It would take precious time from the smallsat community that they need to build their flight articles, and

3. It would be difficult for them to produce a dynamically accurate simulator.

To ensure that what flew is representative of what was analyzed, ULA built and analyzed a mass simulator with a fundamental frequency of over $100 \mathrm{hz}$. This high frequency structure was chosen because it can be considered a stiff mass for analytical purposes and it is very easy to build. Using this approach, ULA ran coupled loads analyses for the mission using both the flight OUTSat finite element model and a stiff mass attached to the ABC plate in lieu of OUTSat. The loads from the two cases were enveloped and provided to both the primary spacecraft and the Launch Vehicle Contractor (LVC) to review. This way, the community is ensured that whatever configuration flies, it has been analyzed and reviewed for acceptability.

\section{ABC Environments and OUTSat Qualification}

The P-PODs and NPSCuL have previously been qualified to the NASA General Environmental Verification Specification (GEVS). However, both the random vibration and thermal environments of the Atlas $\mathrm{V}$ for the $\mathrm{ABC}$ require retesting of both components.

Surprisingly, the thermal environment is not driven by the RL-10 engine nozzle - as it is relatively insignificant. Instead, it is most influenced by initial conditions on the ground. The $\mathrm{ABC}$ is located between two cryogenic stages, the Centaur's liquid hydrogen tank above and the 
Atlas booster's liquid oxygen tank below. Once propellants are loaded on launch day, hot air is blown into the interstage area to keep the Atlas $\mathrm{V}$ avionics and ordnance components at acceptable temperatures. Unfortunately, portions of OUTSat are in the direct flow of this hot air and, therefore, reach a steady state temperature that is relatively high before they even see an increase in temperature due to space effects during flight. The result is maximum P-POD temperatures that exceed previous qualification levels. Considering this, Cal Poly successfully completed thermal-vacuum testing of the PPODs in August of 2011. The NPSCuL aluminum structure did not require thermal testing as its thermal properties show adequate margin.

Although the flight profile of each mission will result in slightly different temperatures, the maximum temperature reached prior to launch, which is the primary factor, will be fairly consistent. On this NROL-36 mission, the estimated maximum temperature at the P-POD surface is approximately $180^{\circ} \mathrm{F}$, exceeding previous qual levels. The Maximum temperature expected for the cubesats during flight is $131^{\circ} \mathrm{F}$, As a result the cubesats have completed a 6 hour $140^{\circ} \mathrm{F}$ thermal bakeout prior to integration.

The random vibration environment levels at the $\mathrm{ABC}$ interface are very severe and exceed those specified in the NASA GEVS as well. This required an additional random vibration qualification test of the NPSCuL structure fully loaded with P-PODs. The assembly was successfully tested in all three axes consistent with the requirements of Military-Standard (MIL-STD)-1540 and provided the test levels required for the individual cubesats. Unfortunately, these levels are extremely high. NPS worked with industry experts to define the lowest appropriate test levels to be specified for the cubesats, but they still resulted in requirements in excess of any that cubesats have been tested to in the past. Based on instrumentation included in the qualification testing, NPS was able to provide P-POD position-specific environments, offering some relief to cubesats in four of the P-PODs, but the worst case positions and axis still resulted in approximately $23 \mathrm{gRMS}$.

It is believed that these qualification levels are conservatively high. Without flight data, the margin provided is prudent for mission assurance reasons. Considering future missions, instrumentation has been added to the ABC/OUTSat structures and one instrumentation cubesat has been integrated to determine actual flight environments to allow the qualification environments to be realistically refined.

For the development of test plans, procedures, and often the actual conduct of the testing itself, ULA engineers were intimately involved to ensure consistency when it

Willcox came time to assess the qualification status of the OUTSat for flight on the Atlas V. Additionally, Aerospace Corporation engineers with years of satellite and launch vehicle test experience were consulted on the test strategies and provided valuable input and feedback.

Each of the cubesats on the manifest passed their vibration proto-qual testing, some taking advantage of the attenuation experienced in their flight configuration by testing in the NPSCuL. Although most successfully passed, some experienced challenges or even significant problems during the testing. While some could be reworked and qualified later, others had to be removed from the manifest.

Once final integration was completed, the entire OUTSat auxiliary payload completed vibration testing at acceptance levels, as the final step in environmental testing. During this phase of testing, the entire integrated system the workmanship of the final flight build was checked. In the case of one cubesat, a discrepancy in the final build was caught. Loose, non-flight screws were found and removed prior to re-test and re-integration of the cubesat for the final run of the integrated AP. Upon completion, OUTSat was readied for shipment to the launch base.

\section{Assembly, Integration \& Test}

Each OUTSat component was assembled and tested by the respective developers. They were also required to complete testing consistent with MIL-STD-1540, and test levels were derived either directly from the Atlas-OUTSat Interface Control Document (ICD) or based on results from OUTSat-level testing. Since many cubesat programs have limited budgets and are one-of-a kind units, a number of the providers chose to follow the requirements for conducting proto-qualification testing on the flight unit. Others fully qualified a "qual unit" and completed the appropriate acceptance testing on the actual flight article.

Because of the harsh vibration environments and the MIL-STD 1540C requirement to drive the vibration testing at the most external common interface of the test configuration, the cubesats had the option to test individually or take advantage of the attenuated levels experienced while mounted in the NPSCuL by testing in an integrated test configuration. As noted above, NPS also provided position dependent levels that resulted in a less extreme environment for those cubesats located in "middle" P-POD's. This allowed those cubesats to test to lower levels as specified for their assigned P-POD position. Once testing was completed, the cubesats were delivered to Cal Poly for integration into OUTSat.

As for testing of the remaining components, each P-POD 
completed acceptance level vibration testing and thermal bake-out; no environmental testing was required on the integrated P-POD/cubesat assembly, as there are only surface to surface contacts without fasteners or electrical interfaces and the combinations were to be tested as part of the integrated OUTSat; the NPSCuL went through a thermal bake-out prior to installation of the integrated PPOD's; and, once fully assembled, the OUTSat completed the required acceptance testing per MIL-STD1540. Figure 5 is a photograph of the completed OUTSat shortly after completing acceptance testing at the Naval Postgraduate School.

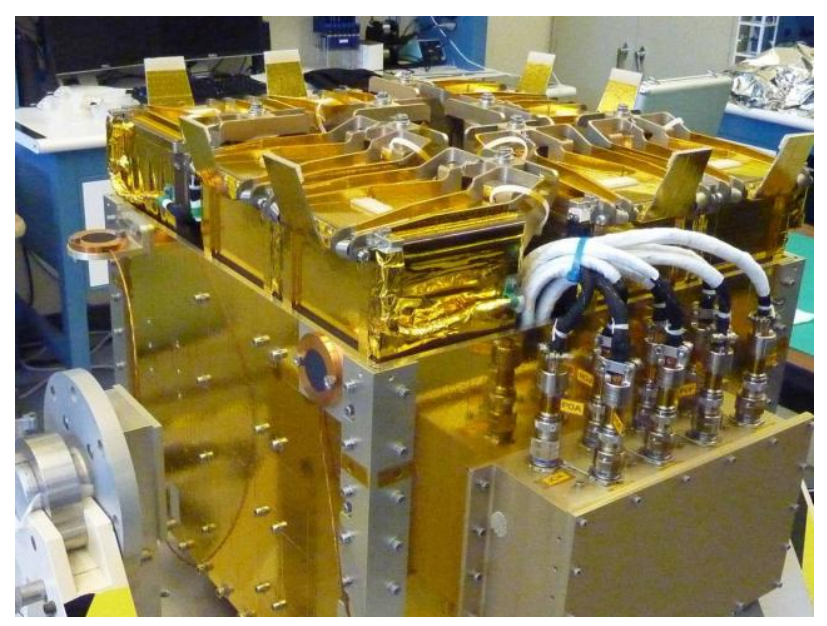

Figure 5: Completed OUTSat

\section{OUTSat Status}

OUTSat has now been mounted onto the ABC plate, together they have been installed on the aft bulkhead of the Centaur upper stage, and the Centaur is stacked on the Atlas V common core booster of NROL-36 on the launch pad, being processed for launch on 2 Aug 2012. To arrive at this point, a monumental achievement, OSL has worked closely and/or contracted with NRO MSD, NASA LSP, ULA, Vandenberg AFB Range Safety, Cal Poly, SRI International, NPS and the CubeSat developers to successfully complete the following:

1. Development of multiple ICD's

2. Verification of over 40 ICD requirements for each cubesat ICD

3. Verification of 81 Launch Vehicle (LV)-OUTSat ICD requirements

4. Multiple Ground Operations Working Groups (GOWGs)

5. Development of all necessary ground operations plans and procedures
6. Submittal of all required Range Safety documentation

7. NPSCuL and P-POD fit checks and qualification testing

\section{OUTSat PDR}

9. Gate 1 review for approval to integrate ABC/OUTSat on NROL-36

\section{OUTSat CDR}

11. Gate 2 review to determine Interim AP Readiness

12. Mission Readiness Reviews for each cubesat

\section{Lessons Learned}

There have been several important lessons gleaned from this initial rideshare effort for the NRO. Some of the most poignant follow:

1. Each smallsat/cubesat with 3 inhibits, providing a dual fault tolerance, for system power on limits any EMI/EMC interference concerns and provides as much flexibility as possible.

2. Work early to identify interdependencies or potential interferences between the primary and secondary payloads (e.g.: anything that would drive the need to have an absolutely final AP manifest, meaning final acceptance testing completed).

3. Establish a nominal auxiliary payload schedule with no later than dates considering all relevant dependencies.

4. Complete Non-Disclosure Agreements between ULA and the AP members early.

5. Ensure early coordination with the range on hazard analysis and safety documentation.

\section{Conclusion}

This effort has provided evidence showing the Aft Bulkhead Carrier is a viable option to place small satellites in orbit using a reliable vehicle that launches on a regular basis. The amount of work is not insignificant, the risks cannot be ignored, but with the right team and proper planning this is an approach the NRO will be proud to use again in the future.

\section{Acknowledgements}

Finally, I would like to thank those who assisted me in this effort specifically. The feedback, input and support I received from my Systems Engineering and Technical 
Advisor (SETA), Dave Callen of Scitor; Leta Jackson of General Dynamics; and Capt Sarah Klaeser-Fernandez of OSL was invaluable to the completion of this paper and the success of this first-of-its-kind rideshare mission.

\section{Biographiy}

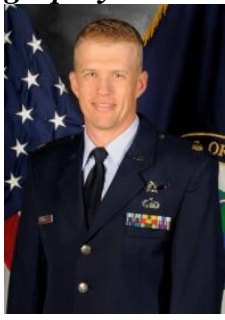

Maj Travis Willcox received a B.S. in Mechanical Engineering from the U.S. Air Force Academy in 2001 and an M.S. in Systems Engineering from the Naval Postgraduate School in 2011. He has spent the last 10 years as a space professional working on Minuteman III ICBM refurbishment, GPS Block IIF System Engineering/AI\&T, deployed in support of Operation Iraqi Freedom and Operation New Dawn, and now as a mission manager in the Office of Space Launch at the National Reconnaissance Office. 